\title{
Amygdala Transcriptome and Cellular Mechanisms Underlying Stress-Enhanced Fear Learning in a Rat Model of Posttraumatic Stress Disorder
}

\author{
Igor Ponomarev ${ }^{*, 1,4}$, Vinuta Rau ${ }^{2,4}$, Edmond I Eger², R Adron Harris' and Michael S Fanselow ${ }^{3}$ \\ 'Waggoner Center for Alcohol and Addiction Research and the College of Pharmacy, University of Texas, I University Station, Austin, TX, USA; \\ ${ }^{2}$ Department of Anesthesia and Perioperative Care, University of California, San Francisco, CA, USA; ${ }^{3}$ Department of Psychology, University of \\ California, Los Angeles, CA, USA
}

Severe stress or trauma can cause permanent changes in brain circuitry, leading to dysregulation of fear responses and the development of posttraumatic stress disorder (PTSD). To date, little is known about the molecular mechanisms underlying stress-induced long-term plasticity in fear circuits. We addressed this question by using global gene expression profiling in an animal model of PTSD, stressenhanced fear learning (SEFL). A total of 15 footshocks were used to induce SEFL and the volatile anesthetic isoflurane was used to suppress the behavioral effects of stress. Gene expression in lateral/basolateral amygdala was measured using microarrays at 3 weeks after the exposure to different combinations of shock and isoflurane. Shock produced robust effects on amygdalar transcriptome and isoflurane blocked or reversed many of the stress-induced changes. We used a modular approach to molecular profiles of shock and isoflurane and built a network of regulated genes, functional categories, and cell types that represent a mechanistic foundation of perturbation-induced plasticity in the amygdala. This analysis partitioned perturbation-induced changes in gene expression into neuronand astrocyte-specific changes, highlighting a previously underappreciated role of astroglia in amygdalar plasticity. Many neuron-enriched genes were highly correlated with astrocyte-enriched genes, suggesting coordinated transcriptional responses to environmental challenges in these cell types. Several individual genes were validated using RT-PCR and behavioral pharmacology. This study is the first to propose specific cellular and molecular mechanisms underlying SEFL, an animal model of PTSD, and to nominate novel molecular and cellular targets with potential for therapeutic intervention in PTSD, including glycine and neuropeptide systems, chromatin remodeling, and gliotransmission.

Neuropsychopharmacology (2010) 35, |402-14II; doi:I0.I038/npp.2010.10; published online 10 February 2010

Keywords: PTSD; fear learning; amygdala; genomics; neurons; astrocytes

\section{INTRODUCTION}

Fear is an adaptive reaction in animals and humans that initiates defensive behaviors in response to cues that signal environmental threats or stressful events (Fanselow and Lester, 1988). For fear to be adaptive, defensive responses must be proportional to the level of threat. Inappropriately regulated responses to threats can lead to negative physical and psychological outcomes. Exposure to an extremely stressful event should normally lead to a proportionate level of fear and coping responses. However, if these fear responses do not subside over time and

*Correspondence: Dr I Ponomarev, Waggoner Center for Alcohol and Addiction Research, University of Texas at Austin, I University Station, A4800, Austin, TX 787I2, USA, Tel: + 512-47I-5I22, Fax: + 512-2322525, E-mail: piatut@mail.utexas.edu

${ }^{4}$ These two authors contributed equally to this work.

Received 14 October 2009; revised 17 December 2009; accepted 18 January 2010 interfere with normal functioning, this may lead to the development of an anxiety disorder such as posttraumatic stress disorder (PTSD) (Rosen and Schulkin, 1998). Hallmark signs of PTSD include re-experiencing the trauma, avoiding stimuli associated with the trauma, numbing of general responsiveness, and symptoms of increased arousal (American Psychiatric Association, 1994). In addition, many PTSD patients show an exaggerated reaction when presented with a mild stressor or things or events that resemble the traumatic stressor (Dykman et al, 1997).

To study the neurobiological mechanisms of this key aspect of PTSD, we used an animal model based on fear conditioning (Rau et al, 2005, 2009; Rau and Fanselow, 2009). In a typical fear conditioning procedure, rats rapidly come to associate an aversive stimulus, such as shock, with a neutral one, such as the environment (context) in which the shock is received. After pairing shock and context, rats will show innate fear responses, such as freezing (immobility), in response to presentation of the context in the 
absence of shock. Freezing serves as an index of fear learning and can be easily quantified (Rau et al, 2005). Typically, freezing increases asymptotically to a maximum as the number of shocks given increases (Fanselow and Bolles, 1979). However, if an animal is exposed to a stressor before fear conditioning, the stressor can enhance the acquisition of subsequent fear learning. For example, rats pre-exposed to a stressor of 15 footshocks in one environment show an enhanced freezing response to a single 'reminder' footshock in a second environment (Rau et al, 2005). This sensitization effect is not limited to the same modality of the stressor. For example, pre-exposure to a series of tailshocks enhances conditioning to periorbital shock (Waddell et al, 2008) or footshock (Baratta et al, 2007) and also increases the fear response to innately aversive stimuli, such as the odor of a predator (Baratta et al, 2007), suggesting that previous stress produces generalized sensitization reaction to mild stressors and perhaps reminders of the previous stress. Several convergent lines of evidence indicate that this increase in fear reflects acquisition of new fear conditioned by the single shock rather than expression of the fear already conditioned by the 15 shocks (Rau et al, 2005). This stress-enhanced fear learning (SEFL) is akin to the disproportionately strong responses that PTSD patients show to reminders of the stressor. Because the exaggerated reaction to a reminder shock can be elicited months after the initial exposure to the intense stressor (Rau et al, 2009), it has been hypothesized that previous trauma produces permanent mechanistic changes in fear-learning circuits.

Neurocircuitry models of PTSD implicate the amygdala as a key structure mediating the effects of stress on the consolidation and recall of traumatic memories (Maier $e t a l$, 1993; Rosen and Schulkin, 1998; Waddell et al, 2008; Christianson et al, 2009). Multiple lines of evidence indicate that acute and chronic stressors induce various forms of structural and functional plasticity in the amygdala, ultimately leading to a long-lasting increase in excitability of principal glutamatergic neurons of the basolateral amygdala, in part, by reducing GABA inhibitory signaling and increasing spinogenesis of the principal neurons (Roozendaal et al, 2009). This shift in balance between inhibition and excitation is hypothesized to provide a mechanistic foundation for the 'hyperresponsive' amygdala, which, in turn, contributes to PTSD symptomatology (Shin and Liberzon, 2009).
To date, little is known about the molecular mechanisms underlying stress-induced plasticity in the amygdala. To identify long-lasting stress-induced molecular changes in the amygdala that underlie the neuroplasticity and SEFL, we used global gene expression profiling and a two-dimensional experimental approach that allowed us to promote and prevent the neurobiological state underlying SEFL. A total of 15 footshocks were used to induce SEFL and a subanesthetic dose of isoflurane, which is a volatile anesthetic that has previously been shown to block SEFL (Rau et al, 2009), was used to suppress the behavioral effects of stress. In all, four experimental groups were compared, in which global gene expression was measured at 3 weeks after the initial exposure to different combinations of shock and isoflurane. Our hypothesis was that the intense stressor of 15 footshocks would produce prolonged changes in gene expression, whereas isoflurane would suppress shockinduced changes of critical genes required for the regulation of brain plasticity and behavior. This study validated known and identified novel molecular and cellular targets with potential for therapeutic intervention in PTSD.

\section{MATERIALS AND METHODS}

\section{Animals}

The protocol for this study was approved by the UCSF institutional animal care and use committee and all animal treatments were carried out at UCSF. A total of 56 adult male Long Evans rats (Harlan; Indianapolis, IN), 70-90 days old, were used in this experiment. The rats were housed 2-3 per cage in the UCSF animal care facility, in which food and water were available ad libitum before and during the experiment. The animals were kept on a $12: 12$-h light-dark cycle, with lights coming on at $0600 \mathrm{~h}$. All experimental procedures took place during the light cycle. The rats were handled daily for a week before the experiment. Handling consisted of picking up the rat and holding it for approximately $20 \mathrm{~s}$. Handling serves to decrease baseline anxiety levels.

\section{Experimental Design}

Experimental design for this study is summarized in Table 1. On day 1, rats were transported to an experimental room and placed in an equilibration chamber in which they

Table I Experimental Design

\begin{tabular}{|c|c|c|c|c|c|c|}
\hline Day I context A & & $n$ & Day 22 & $\begin{array}{c}\text { Day } 23 \\
\text { context B }\end{array}$ & $\begin{array}{c}\text { Day } 24 \\
\text { context B }\end{array}$ & $\begin{array}{c}\text { Day } 25 \\
\text { context A }\end{array}$ \\
\hline $0 \%$ Isoflurane & 0 shocks & 8 & 30 min in context B & I shock & Test & Test \\
\hline $0 \%$ Isoflurane & I5 shocks & 8 & 30 min in context B & I shock & Test & Test \\
\hline 0\% Isoflurane & 15 shocks & 6 & Amygdala extracted & - & - & - \\
\hline $0.6 \%$ Isoflurane & I5 shocks & 8 & 30 min in context $B$ & I shock & Test & Test \\
\hline $0.6 \%$ Isoflurane & 15 shocks & 6 & Amygdala extracted & - & - & - \\
\hline
\end{tabular}


received 0 or $0.6 \%$ isoflurane exposure for $30 \mathrm{~min}$. After equilibration, animals were placed into one of four fear conditioning chambers in 'context A', which were filled with an isoflurane concentration equivalent to the concentration in the equilibration chamber. This dose of isoflurane was chosen because it blocks SEFL (Rau et al, 2009) without completely sedating animals. Footshocks in rats pretreated with this dose elicit activity bursts that are qualitatively similar to untreated rats. Rats were randomly assigned to either receive 0 or 15 shocks for a total exposure time of $93 \mathrm{~min}$. After 3 weeks (day 22), a random half of the animals in each group were decapitated and the lateral/basolateral amygdaloid complex removed. The tissue samples were stored at $-70{ }^{\circ} \mathrm{C}$ overnight and sent the following day to the UT Austin for gene array analysis. The other half of the animals were assigned to the behavioral control experiment. On day 22, these rats were habituated to 'context B' for $30 \mathrm{~min}$ to reduce any baseline freezing. The following day (day 23), all behavioral control rats were placed in context B and given a single shock. The next day (day 24), rats were returned to context $\mathrm{B}$ for $8 \mathrm{~min}$ and $32 \mathrm{~s}$ and tested for fear conditioned to the B context. The following day (day 25), rats were returned to context $\mathrm{A}$ for $8 \mathrm{~min}$ and $32 \mathrm{~s}$ and tested for fear conditioned to the A context. Freezing, a measure of fear, was scored during each context test and a percentage of freezing was calculated. All behavioral experiments were conducted by the same experimenter. Analysis of variance (ANOVA) followed by Fisher's PLSD test was used to analyze freezing. Detailed experimental design is presented in Supplementary Information.

To minimize the effects of potential reminders of the original fear conditioning on gene expression, much care was taken to ensure that all procedures on the day of killing were different from those performed on day 1, when the 15-shock stressor was given. Rats were transported differently and housed in a different space within the lab. Animals were not handled between the shock day and the day of killing and the killing was performed in a different room than the conditioning room.

\section{Follow-Up Validation Experiment}

In all, 22 adult male Long Evans rats were assigned to one of three groups: 0 shocks $+0 \mathrm{mg} / \mathrm{kg}$ etomidate $(n=8), 15$ shocks $+0 \mathrm{mg} / \mathrm{kg}$ etomidate $(n=7)$, or 15 shocks $+40 \mathrm{mg} / \mathrm{kg}$ etomidate $(n=7)$. The procedure was conducted as previously described, with few exceptions. Rats were given an intraperitoneal injection of either $0 \mathrm{mg} / \mathrm{kg}$ etomidate or $40 \mathrm{mg} / \mathrm{kg}$ etomidate and placed back in their homecage. Etomidate (Chem Pacific, Baltimore, MD) was mixed in $70 \%$ dimethyl sulfoxide (Sigma-Aldrich, St Louis, MO) and sterile saline solution (Hospira, Lake Forest, IL). After $30 \mathrm{~min}$, rats were transported to the fear conditioning chambers and given 0 or 15 shocks. All groups were given the single shock the following day (day 2), the test in context $\mathrm{B}$ on day 3 , and the test in context $\mathrm{A}$ on day 4 .

\section{Microarray Analysis and qRT-PCR}

Total RNA was isolated from bilateral amygdalae from individual animals (one animal per sample) and the samples were sent to Asuragen, Austin, Texas, where mRNA was amplified, labeled, and hybridized on Illumina RatRef-12 Expression BeadChip microarray platform containing $\sim 22500$ known genes and ESTs. Before hybridization, the samples from different treatment groups were counterbalanced on each chip to avoid batch effects. Transcript abundance was measured by fluorescent intensity after scanning. Several genes were selected for technical validation using RT-PCR. RT-PCR was conducted using total RNA (before amplification) from the same samples used for microarray experiments. All real-time TaqMan assays are pre-designed by Applied Biosystems (Foster City, CA) and labeled with FAM as a reporter and a nonfluorescent quencher. Detailed TaqMan protocols are available on the manufacturer's website: http://www.appliedbiosystems. com/index.cfm.

\section{Modular Approach}

To detect genes and functional groups important for neuroplasticity and SEFL, we used a two-step modular approach. This approach is based on recent literature showing that (1) genes are not regulated in isolation and transcriptomes are organized into modules of coexpressed (correlated) genes and (2) many modules of coexpressed genes reflect functional and structural organization of brain tissue and can be explained by known biological concepts, such as genomic location, functional group, or cell type (Oldham et al, 2008; Ge et al, 2003). In the first step, all data were analyzed using ANOVA, false discovery rate (FDR) was estimated and significant genes were selected for the second step. Because this approach does not assume independence of each expression variable (gene), a higher FDR can be tolerated. In the second step, significantly regulated genes were clustered into modules based on geneby-gene correlations across all samples. Organizing principles and biological significance of each module were then assessed through functional over-representation (enrichment) analysis and an overall significance of individual genes was decided based on a combination of its statistical significance and biological relevance of its module.

\section{Statistics and Informatics}

Data for each gene were analyzed using two-way ANOVA, with Shock and Isoflurane as between-subject factors. In addition, the group treated with $0 \%$ isoflurane and 15 footshocks, which is the only group showing SEFL, was compared with the other three groups using planned comparisons (contrast ANOVA) to identify genes associated (correlated) with SEFL. Only genes detected (detection $P<0.1)$ in at least three subjects per treatment group were analyzed. Normalization was performed using a $Z$-score transformation of log base 2 background-subtracted values. Before statistical analysis, outlier values for each gene within a treatment group and one outlier array were removed based on Grubbs' test. Nominal $P$-values for each factor (main effect of isoflurane, main effect of shock, and association with SEFL) were compared with a distribution of $P$-values based on 100 random permutations of samples to obtain permutation-based $P$-values. This analysis was followed by an FDR analysis to estimate the percentage of false positives. Free-access $Q$-value software (Storey, 2002) 
was used to calculate the $Q$-value (a measure of FDR) for each transcript. Significant genes $(P<0.05 ; Q<0.3)$ for at least one of the factors examined were further analyzed. Data for significantly regulated genes were normalized within each group using a $Z$-score to obtain 'innate' correlations unbiased by the treatments and the three gene lists were subjected to K-mean clustering (STATISTICA 6.1; Statsoft, Tulsa, OK) to identify clusters of coexpressed genes. Genes from each cluster were then subjected to functional group over-representation analysis (see Supplementary Information for details) using the Database for Annotation, Visualization and Integrated Discovery (DAVID, Huang et al, 2007) and cell type-specific data sets (Cahoy et al, 2008) to analyze how genes in each cluster are linked (function, structure, transcriptional regulation, and cellular localization). Gene symbols of significantly regulated genes from each cluster were converted to GeneBank Accession IDs and uploaded to DAVID as gene lists. All genes on array that passed the detection filter (11 480) were used as a background list. Before conversion, suffixes '_predicted' or '_mapped' were deleted to assist with proper identification in DAVID. Cell typespecific data sets were downloaded in MS Excel format from the Journal of Neuroscience website (Cahoy et al, 2008). Genes with at least fourfold enrichment in either neurons, astrocytes, or oligodendrocytes were used to assign cell type specificity to significantly regulated genes. Cell type-specific over-representation analysis for each cluster was carried out using a hypergeometric distribution (http://stattrek.com/ Tables/Hypergeometric.aspx). Identification of over-represented functional groups provides an additional level of statistical validity that mitigates effects of moderate falsepositive rates for individual genes. An enrichment $P$-value generated by a hypergeometric test is assigned to each group and FDR is estimated. In addition, hypergeometric distribution and $\chi^{2}$ tests were used to compare gene frequencies whenever appropriate.

\section{RESULTS}

\section{Fear Behavior}

When compared with the other three groups, animals given 15 shocks and $0 \%$ isoflurane (SEFL group) showed a conditional fear response when tested in context A on day 25 and an enhanced fear conditioned to the single shock (SEFL) when tested for fear in context B on day 24 (Figure 1). Isoflurane at $0.6 \%$ given during administration of the 15 shocks 3 weeks earlier suppressed both conditional fear to the 15 shocks and SEFL. In summary, exposure to a severe stressor of 15 footshocks in context A produced longlasting biological changes associated with a robust enhancement of fear learning in context B. These behavioral results replicate our previous findings (Rau et al, 2009) and provide a validated behavioral counterpart for rats used in the gene array analysis.

\section{Global Changes in Gene Expression}

Both shocks and isoflurane produced global changes in gene expression (Supplementary Information: Supplementary Figure S1). In this study, 'Global' refers not only to the
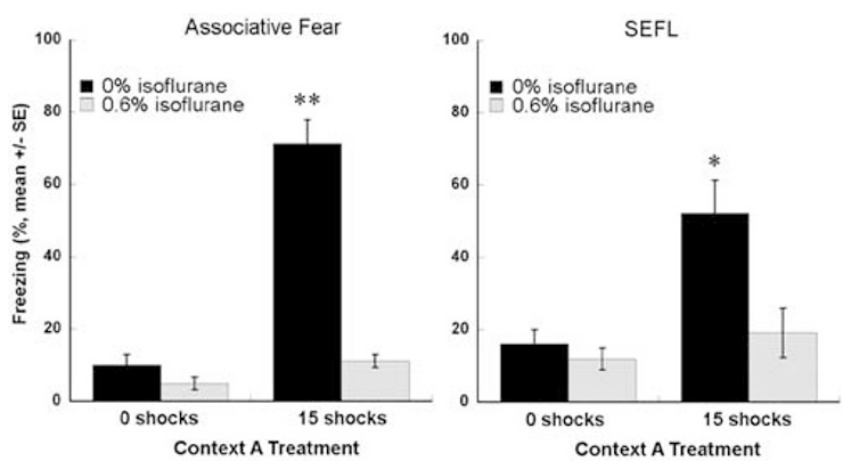

Figure I Fear conditioning responses measured on day 25 in contexts $A$ (left panel) and on day 24 in context B (right panel). The two-way ANOVAs showed significant effects of shock, isoflurane, and an interaction between the two factors (All $P<0.05$ ). The asterisks indicate significant difference between the 15 shock $/ 0 \%$ isoflurane group and each of the other three groups $(* P<0.01$; $* * P<0.00 I)$.

number of transcripts measured simultaneously, but also to the number of significantly changed genes under a certain condition. The number of genes regulated at $P<0.05$ was considerably greater $\left(\chi^{2} P<1 \mathrm{E}-10\right)$ than one expected by chance, indicating a profound effect of environmental challenges on the amygdalar transcriptome. Several genes were regulated by both shock and isoflurane, and the number of genes regulated in opposite directions by the two factors greatly exceeded the number of genes in the same direction (Supplementary Information: Supplementary Figure S2; $\left.\chi^{2} P<1 \mathrm{E}-10\right)$, suggesting that isoflurane can reverse or counterbalance the effects of shock on gene expression. These contrasting effects of the two variables appear to contribute to patterns in gene expression consistent with the separation of the SEFL group from the other three. Contrast ANOVA showed that the number of genes regulated at $P<0.05$ was indeed significantly greater $\left(\chi^{2} P<1 \mathrm{E}-10\right)$ than expected by chance, indicating that SEFL is associated with global changes in gene expression (Supplementary Information: Supplementary Figure S1C). Overall, 3395 transcripts were affected by either shock and/ or isoflurane and/or associated with SEFL. This combined gene list is referred to as the regulatome.

We next explored the relationships among genes affected by each of the three variables using cluster analysis and analyzed functional significance of each cluster using gene annotations and over-representation (enrichment) analysis. Many genes were shared among different clusters affected by one of the three factors, and this allowed us to identify significantly overlapping clusters and build modules of overlapping clusters that share biological determinants. Results of K-mean clustering and over-representation analyses are summarized as a meta-network of overlapping clusters and corresponding highly over-represented functional and structural categories (Figure 2). Lists of all significantly regulated genes, their cluster association, and over-represented functional groups are given in Supplementary Tables S1 and S2.

The amygdala regulatome was organized into modules of coexpressed (correlated) genes and gene clusters that corresponded to cell types and functional groups. Among highly enriched functional categories were groups and 


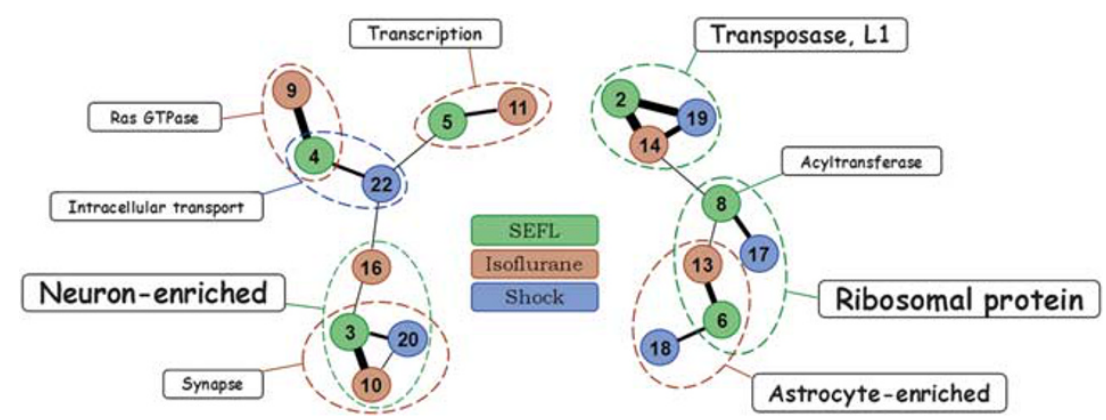

Figure 2 A meta-network of amygdalar regulatome shows top results of K-mean clustering and functional group over-representation analysis. Gene clusters are numbered as I-24 (SEFL: I-8; isoflurane: 9-16; and shock: 17-24). Only clusters linked to a highly over-represented functional group or cell type $(P<0.000$ I; FDR $<1 \%)$ are shown and only significantly overlapping clusters $\left(\chi^{2} P<0.005\right.$; at least $10 \%$ overlap) are connected. Encircled are modules of overlapping clusters linked to a highly over-represented functional group, which is over-represented to some degree in each cluster of a given module. Color of the circle and the line connecting modules and over-represented groups corresponds to the color of the most significant cluster in the module. Thickness of lines between clusters and font sizes are proportional to the percentage of overlap and significance level of over-represented groups, respectively.

pathways previously implicated in brain plasticity, such as synaptic transmission, transcription factors (see Supplementary Information: Supplementary Figure S3 for details), and Ras GTPases. Examples of individual genes involved in regulation of synaptic plasticity and memory formation include brain-derived neurotrophic factor, $B d n f$, and cAMP responsive element binding protein 1, Creb1 (Dennis and Levitt, 2005; Chwang et al, 2007; Lubin et al, 2008). This analysis also revealed novel functional categories not usually implicated in brain plasticity, such as LINE-1 (L1) type transposase domain-containing genomic elements. Transcripts representing L1 transposable elements were upregulated by shock and downregulated by isoflurane, resulting in cumulative upregulation in the SEFL group when compared with the other three (see Supplementary Information: Supplementary Figure S4 for details). Of particular interest were two modules of clusters that included genes enriched in either neurons or astrocytes.

\section{Cell Type-Specific Changes in Gene Expression}

Our network analysis clearly partitioned perturbationinduced changes in gene expression into neuron- and astrocyte-specific changes (Figure 3). There were 144 neuron-enriched genes and 84 astrocyte-enriched genes regulated by one or more factors. Shock and isoflurane produced contrasting effects on transcriptomes of these cell types; most neuron-enriched genes were downregulated by shock and upregulated by isoflurane, whereas genes enriched in astroglia were mainly downregulated by isoflurane and upregulated by shock (Supplementary Information: Supplementary Figure S5). SEFL behavior was negatively correlated with most neuron-enriched genes (downregulation in the SEFL group) and positively correlated with most astrocyte-enriched genes (upregulation in the SEFL group) (Figure 3 and Supplementary Information: Supplementary Figure S5). These contrasting effects in different cell types suggest coordinated transcriptional responses to treatments in neurons and astrocytes. This finding was further validated by examining correlations between neuron- and astrocyte-enriched genes. The number of pairwise correlations at $P<0.01$ was 900 , more than eight times greater than one expected by chance $\left(\chi^{2} P<1 \mathrm{E}-100\right)$. Furthermore, $87 \%$ of these correlations were negative ( $\chi^{2} P<1 \mathrm{E}-100$ compared with $50 \%$ chance), suggesting that molecular responses to perturbations in neurons and astrocytes are tightly linked. The finding that transcriptional networks in neurons and astrocytes are negatively correlated was noted earlier (Oldham et al, 2008), a puzzling observation that warrants further investigation. As a first step, we built a network of highly correlated cell typespecific genes that should be useful in generating specific hypotheses as to the relationships between molecular networks of neurons and astroglia (Supplementary Information: Supplementary Figure S6). Figure 3 shows several cell type-specific genes with previously determined roles in the brain's normal functions and in brain abnormalities. The presence of the vesicular glutamate transporter 2, VGLUT2 (Slc17a6), and the GABA vesicular transporter, VGAT (Slc32a1), suggested that molecular plasticity in the amygdala occurred in both principal glutamate neurons and GABA interneurons.

\section{'Drugable' Gene Targets}

Because of the need for new pharmacotherapies for PTSD, genes representing drugable targets are of particular interest. We found four groups of neurotransmitter/ neuromodulator systems that had at least two key members associated with SEFL: (1) Glutamate, represented by the NMDA receptor 3b subunit (Grin3b), serine racemase (Srr), VGLUT2 (Slc17a6), and glial high affinity glutamate transporter, EAAT1 (Slc1a3); (2) GABA, represented by three GABAA receptor subunits (Gabrb2, Gabrb3, and Gabra4) and VGAT (Slc32a1); (3) Glycine, represented by two glycine receptor subunits (Glra2, Glrb); and (4) Neuropeptides, represented by several neuropeptides and somatostatin receptor type 2 (Sst, Sstr2, Npy, Crhbp, Bdnf, Adcyap1 (PACAP), Egf). Glutamate and GABA systems have been implicated in regulation of stressinduced neuroplasticity and fear learning, but glycinergic neurotransmission and modulation by neuropeptides, such as somatostatin, represent novel targets for PTSD treatment. 

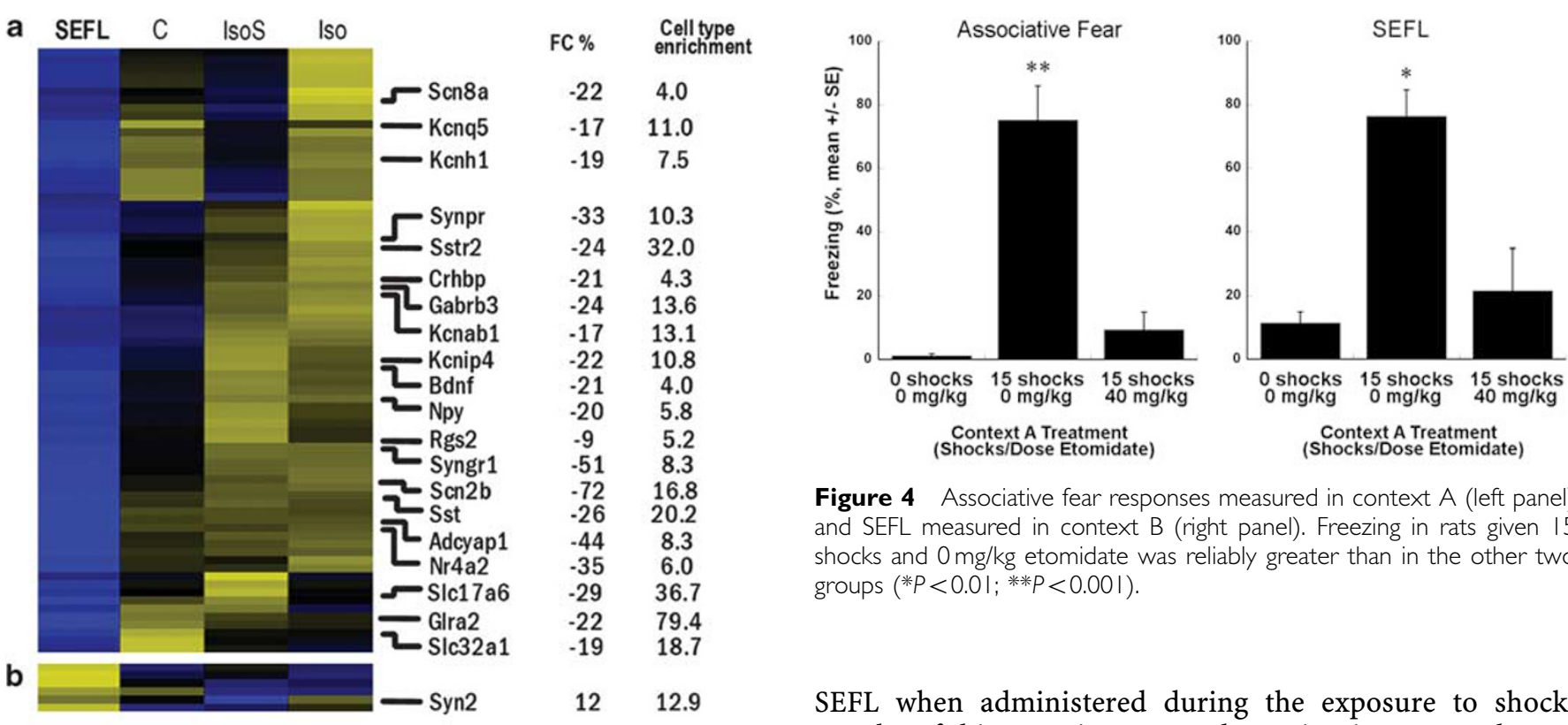

Figure 4 Associative fear responses measured in context A (left panel) and SEFL measured in context B (right panel). Freezing in rats given 15 shocks and $0 \mathrm{mg} / \mathrm{kg}$ etomidate was reliably greater than in the other two groups $(* P<0.01$; $* * P<0.001)$.

\section{c}

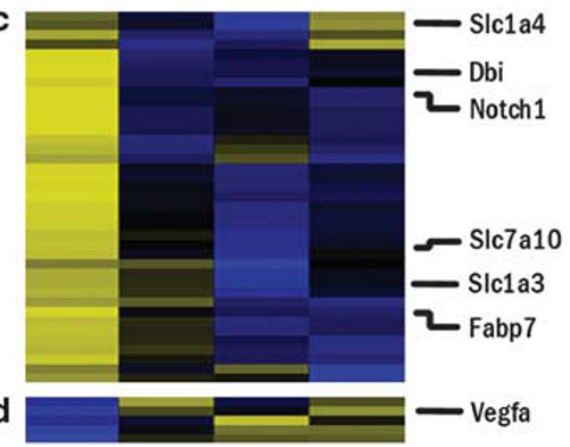

22

10.7

12

4.7

4.4

12

27.5

$10 \quad 43.0$

15

6.2

$-26$

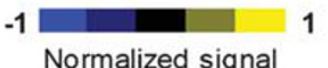

Normalized signal
Figure 3 Expression patterns of genes enriched in neurons $(a, b)$ or astrocytes (c, d) and associated with SEFL behavior. Cell type- specific genes were identified based on literature (Cahoy et al, 2008). A clustergram of centered and normalized group means is shown. SEFL = SEFL group; $C=$ control group ( $0 \%$ isoflurane, 0 shocks); IsoS $=0.6 \%$ isoflurane, 15 shocks; Iso $=0.6 \%$ isoflurane, 0 shocks. Gene symbols of several genes implicated in brain functions are shown to the right of the clustergram. FC\% $=$ percent fold change in the SEFL group compared with the average of the other three groups. Cell-type fold enrichment is shown on the right. Clustergram was created using Cluster 3.0 and Java TreeView I.I.3.

\section{Validation of Microarray Results}

Our microarray experiment generated several hypotheses that could be tested by means of behavioral pharmacology. For example, three GABAA receptor subunits (Gabrb2, Gabrb3, and Gabra4) and the GABA vesicular transporter, VGAT (Slc32a1), were negatively correlated with SEFL, that is, downregulated in the SEFL group when compared with the other three groups, suggesting a role of reduced GABA signaling in mechanisms of SEFL. We conducted an experiment to show a proof of principle that our microarray results can be validated at a systems level. We hypothesized that etomidate, an allosteric modulator selective for $\beta 2$ or $\beta 3$ subunit-containing GABAA receptors, will decrease

\section{DISCUSSION}

Defensive responses to threats are evolutionarily adaptive, but inappropriate regulation of fear can lead to negative physical and psychological outcomes such as PTSD. PTSD is characterized by an exaggerated emotional reaction to mild stressors. This key aspect is modeled in rats showing enhanced contextual fear (SEFL) to a mild reminder stressor after experiencing a severe stressor (Rau et al, 2005). Our model produces two potentially dissociable effects. One effect is associative fear conditioning to the different cues present in each environment. The second effect is a permanent non-associative change in the neurobiological mechanisms that mediate future responses to stressors (ie, SEFL). Associative fear to the reminder shock is enhanced in pre-shocked animals due to a non-associative mechanism. We used global gene expression profiling to identify individual molecules and cellular functions that may represent this non-associative mechanism. Our behavioral experiments confirm and extend previous findings (Rau et al, 2009), showing that an enhanced fear response (SEFL) persisted for 3 weeks and a subanesthetic dose of isoflurane during the exposure to 15 shocks suppressed it. The preventive effects of the drug on the formation of traumatic memories provided a validated behavioral counterpart for the microarray analysis. 
We found that 15 shocks produced long-term changes in the amygdalar transcriptome and propose that some of these changes are required for the neuroplasticity that underlies SEFL. We used isoflurane to refine the search for critical genes and tested the hypothesis that isoflurane suppresses changes in gene expression required for neuroplasticity. However, isoflurane did not simply block shock-induced molecular changes but rather produced discrete transcriptional responses that were reliably detected at 22 days after exposure to the drug. A key finding was that most genes significantly changed by both 15 -shock stressor and isoflurane were regulated in opposite directions by the two factors. This finding at the molecular level is consistent with the acute effects of these two factors on neuronal activity. Shock activates a subset of amygdalar neurons during fear conditioning (Reijmers et al, 2007), whereas isoflurane produces general inhibition of neuronal activity and an overall reduction of neuronal excitability (Ranft et al, 2004). Neuronal activity and gene expression are tightly linked through activity-dependent transcription involved in regulation of neuroplasticity (Flavell and Greenberg, 2008). Divergent modulation of neuronal activity by shock and isoflurane could trigger long-term changes in gene expression, with some of these changes offsetting each other to prevent downstream alterations in structure and/or function. Because shock and isoflurane may affect different subpopulations of amygdalar neurons, future studies should focus on dissecting the effects of these two factors at the single neuron level.

To identify molecular mechanisms underlying SEFL, we focused on transcriptional changes that were associated with behavioral differences that were up- or down-regulated in animals that received $0 \%$ isoflurane and 15 shocks (SEFL group) when compared with the other three groups. The contrasting effects of shock and isoflurane contributed to the gene expression patterns, in which animals that developed SEFL were significantly different from the rest. Our systemic multidimensional approach allowed us to build a network of regulated genes and functional categories that represents a mechanistic foundation of perturbationinduced plasticity in lateral/basolateral amygdala, with genes and functional groups associated with SEFL being an integral part of this network. Our further discussion focuses on key findings from this analysis.

\section{Cellular Resolution of Brain Plasticity}

Many regulated genes in amygdala were clustered according to their specific expression in one of the major cell classes. Genes generally enriched in neurons or astrocytes (Cahoy et al, 2008) formed two independent modules in our gene network. These results are consistent with recent literature showing that (1) individual populations of neurons and glial cells are characterized by unique transcriptional signatures (Sugino et al, 2006; Cahoy et al, 2008) and (2) these signatures can be reliably identified from analysis of complex brain tissue without isolating homogeneous populations of cells (Ponomarev et al, 2006; Oldham et al, 2008). Our data suggest coordinated transcriptional responses to homeostatic perturbation in different cell types and point to individual genes and functional categories involved in regulation of brain plasticity.
Previous research implicated several neurotransmitters, neuromodulators, and hormones in stress-induced neuroplasticity in the amygdala and memory consolidation, including glutamate, GABA, noradrenaline, serotonin, and CRH systems (see Galvez et al, 1996; Amat et al, 1998; Roozendaal et al, 2009 for review). A general consensus is that a reduced GABA inhibitory tone and enhanced glutamate and $\mathrm{CRH}$ signaling underlie stress-induced pathological neuroadaptations associated with PTSD symptoms (Roozendaal et al, 2009). Our microarray and behavioral data validated and extended previous findings, suggesting that glycine neurotransmission and modulation by neuropeptides, such as somatostatin, also have an important role. The glycine receptor $\alpha 2$ subunit is the predominant $\alpha$-subunit in the basolateral amygdala, which in combination with the $\beta$-subunit can form functional channels (McCool and Farroni, 2001). Both of these subunits were downregulated in the SEFL group, suggesting that a decrease in glycine inhibition may contribute to an overall reduction in inhibitory tone in the 'hyperresponsive' amygdala. Downregulation of somatostatin and its type 2 receptor in the SEFL group implicate this neuropeptide in mechanisms of SEFL. Somatostatin is a marker of one type of GABA interneurons (Sugino et al, 2006) and may colocalize with several other peptides, including NPY and CRHBP (Smith et al, 1997; Sosulina et al, 2006). Because all three peptides are downregulated in the SEFL group, we hypothesize that severe stress produces a long-term decrease in activity and/or numbers of these neurons, which results in a reduced GABA tone and 'disinhibition' of $\mathrm{CRH}$ signaling. Although specific agonists for $\alpha 2$-containing glycine receptors or somatostatin type 2 receptors are yet to be identified, less-specific compounds that potentiate these receptors can be used in animal models to examine the involvement of glycine transmission and somatostatin modulation in mechanisms of SEFL.

Stress-induced plasticity in the amygdala has been viewed primarily as a result of interactions among various inhibitory, excitatory, and modulatory neuron-based processes (Roozendaal et al, 2009). Our data strongly suggest the involvement of astroglial functions in amygdalar plasticity. Astrocytes have active roles in brain physiology. They communicate with neurons by responding to a number of neurotransmitters and releasing glutamate (Volterra and Meldolesi, 2005). Our results indicate that this key astroglial function is modulated in the amygdala by environmental challenges. For example, an upregulation of the glial high-affinity glutamate transporter EAAT1 (Slc1a3) and downregulation of serine racemase (Srr) in SEFL group is consistent with an enhanced participation of astrocytes in glutamate neurotransmission and modulation. Possible participation of amygdalar astrocytes in mechanisms of traumatic memories suggests astroglial signaling as a novel target for medication development in PTSD. Previous studies suggested an important role of astrocytes in the pathology of some neurodegenerative disorders, such as Alzheimer's disease (Volterra and Meldolesi, 2005) and multiple sclerosis (De Keyser et al, 2004), and medical interventions targeting astroglial functions are being developed. One possibility is to influence the production and release of astrocyte-specific 'gliotransmitters', such as D-serine 
and homocysteic acid, both exerting an effect at the glutamatergic NMDA receptors.

\section{Regulation of Transposons and Role of Chromatin Remodeling}

One surprising finding of our network analysis was regulation of transcripts containing L1 type transposase domain elements. Transposable elements of various classes constitute a large fraction of most eukaryotic genomes, although their function is poorly understood. They are normally silenced by epigenetic mechanisms, including modifications of histone tails, DNA methylation, and alterations in chromatin packing and condensation (see Slotkin and Martienssen, 2007 for review). In plants and animals, environmental stress can release epigenetic silencing, in part, by inhibition of chromatin-mediated gene-silencing mechanisms in the genome (Slotkin and Martienssen, 2007). Several recent publications reported that neuronal genomes can be modified by de novo L1 retrotransposition events (Muotri et al, 2005; Coufal et al, 2009). Although it remains to be determined whether de novo L1 retrotransposition has an active role in stressinduced plasticity, a more plausible explanation for the increased expression of transposable elements in SEFL group is a 'passive' release of chromatin-mediated gene silencing. Epigenetic chromatin remodeling was proposed as a central mechanism for regulation of gene expression during memory formation and development of neurodegenerative disorders (Abel and Zukin, 2008; Roth and Sweatt, 2009). Histone deacetylases (HDAC) are important modulators of chromatin structure. Abel and Zukin (2008) suggested HDAC inhibitors as potential therapeutics for a wide range of neurodegenerative disorders, including Huntington's and Parkinson's diseases. Our data provide a rationale for extending these studies to PTSD.

\section{Isoflurane and the Amygdalar Transcriptome}

The long-lasting effects of isoflurane on global gene expression in the amygdala reported in this study raise an important question with clinical relevance: What are longterm consequences of surgical anesthesia? Although negative effects of anesthetics on immature brain have been reported (Loepke et al, 2009), evidence of the long-term effects of anesthesia in adults is inconsistent. Because the amygdala is the key structure regulating emotionally relevant events, the isoflurane-induced changes in amygdalar transcriptome suggest that surgical anesthesia changes the balance in neurocircuits mediating emotionally charged behaviors, which may have yet-unknown physiological and behavioral consequences. In addition, our finding that isoflurane reversed many shock-induced changes in gene expression offers an intriguing possibility that pretreatment with isoflurane is protective against effects of stress on amygdala and fear learning. However, it is unlikely that isoflurane-induced transcriptional changes prevent subsequent stress-induced plasticity and SEFL, as isoflurane given after the 15-shock stressor did not suppress SEFL (Rau et al, 2009). Most likely, amnesic effects of anesthetics are produced at a whole cell level when applied simultaneously with shock; thus, offsetting shifts in neuronal activity and preventing downstream changes in gene expression. It is possible that isoflurane-induced changes in gene expression increase cellular thresholds to stress, suggesting that a greater number or intensity of shocks may be required to achieve the same level of SEFL, a hypothesis that can be tested in future studies. Another potentially interesting future direction may emerge from our finding that several traditionally termed 'inducible' transcription factors, such as Fos, JunB, Egrl, and Egr2, show transcriptional changes long after the original treatment with isoflurane. The upregulation of the Egr2 gene after isoflurane was reported previously (Rampil et al, 2006), but a much larger dose of the drug and a shorter time point $(6 \mathrm{~h})$ were used. These long-term effects of isoflurane on immediate early genes are intriguing and deserve further investigation.

\section{Behavioral Validation}

Our behavioral validation experiment with etomidate suggested the specific role for $\beta 2$ or $\beta 3$ subunit-containing GABAA receptors in stress-induced plasticity in the amygdala. An alternative explanation of the suppressing affects of isoflurane and etomidate on SEFL is that any general anesthetic would block formation of memory with a large enough dose. However, the subanesthetic concentration of isoflurane that we used to block SEFL does not suppress fear conditioning to a tone (Dutton et al, 2001). Similarly, a moderate dose of the anesthetic agent midazolam, which is a GABAA agonist at the benzodiazepine site, suppresses associative learning to 15 shocks, but not SEFL (Rau et al, unpublished results). These data validate specificity of individual anesthetics in memory processes and suggest that SEFL suppression is not a general effect of all GABAA agonists.

\section{Associative $v s$ Non-Associative Fear}

In addition to the enhancement of fear learning in context B, our SEFL group shows robust associative fear in context $\mathrm{A}$, which raises the concern that some changes in gene expression were triggered by contextual reminders of the original fear conditioning, such as animal handling or the same experimenter. Although the possibility of cueinduced gene expression cannot be ruled out, we made every attempt to minimize the effects of overt memory retrieval on gene expression; animals were habituated to handling before conditioning and killed quickly after they were transferred to the procedure room on day 22 . Moreover, in our behavioral experiment, previously shocked animals did not show more freezing when they were first exposed to context B on day 23 before a single shock was given, suggesting that handling by the same experimenter is not a cue reminder. Several previous studies analyzed molecular mechanisms of the associative component of fear learning using microarrays (Mei et al, 2005; Keeley et al, 2006; Lamprecht et al, 2009). Our study augmented this line of research with focusing on long-term non-associative effects of stress and linking the molecular and cellular changes with PTSD-like symptoms in rats. 


\section{Summary}

This study is the first to use a novel model of PTSD and global gene expression profiling to define molecular and cellular determinants of perturbation-induced plasticity in the amygdala. Environmental challenges produced robust long-lasting effects on amygdalar transcriptome, which reflected functional and structural changes in neurons and astroglia, which are necessary for plasticity and SEFL. Our modular network approach allowed us to estimate functional values of individual genes in the context of their relationships with other genes, their known or predicted physiological role, and their cellular identity, and to formulate refined hypotheses. Our data offer novel molecular and cellular targets with potential for therapeutic intervention in PTSD.

\section{ACKNOWLEDGEMENTS}

We thank Elizabeth Kahanek and Irene Oh for valuable technical assistance and Dr Jim Sonner for helpful discussion. This research was supported by the National Institute of Health Grants GM47818 (to RAH and EIE) and NIMH62122 (to MSF).

\section{DISCLOSURE}

The authors declare no conflict of interest.

\section{REFERENCES}

Abel T, Zukin RS (2008). Epigenetic targets of HDAC inhibition in neurodegenerative and psychiatric disorders. Curr Opin Pharmacol 8: 57-64.

Amat J, Matus-Amat P, Watkins LR, Maier SF (1998). Escapable and inescapable stress differentially alter extracellular levels of $5-\mathrm{HT}$ in the basolateral amygdala of the rat. Brain Res 812: 113-120.

American Psychiatric Association (1994). Diagnostic and Statistical Manual of Mental Disorders, IV edn. American Psychiatric Press: Washington, DC.

Baratta MV, Christianson JP, Gomez DM, Zarza CM, Amat J, Masini CV et al (2007). Controllable versus uncontrollable stressors bi-directionally modulate conditioned but not innate fear. Neuroscience 146: 1495-1503.

Cahoy JD, Emery B, Kaushal A, Foo LC, Zamanian JL, Christopherson KS et al (2008). A transcriptome database for astrocytes, neurons, and oligodendrocytes: a new resource for understanding brain development and function. J Neurosci 28: 264-278.

Christianson JP, Ragole T, Amat J, Greenwood BN, Strong PV, Paul ED et al (2009). 5-Hydroxytryptamine 2C receptors in the basolateral amygdala are involved in the expression of anxiety after uncontrollable traumatic stress. Biol Psychiatry 67: 339-345.

Chwang WB, Arthur JS, Schumacher A, Sweatt JD (2007). The nuclear kinase mitogen- and stress-activated protein kinase 1 regulates hippocampal chromatin remodeling in memory formation. J Neurosci 27: 12732-12742.

Coufal NG, Garcia-Perez JL, Peng GE, Yeo GW, Mu Y, Lovci MT et al (2009). L1 retrotransposition in human neural progenitor cells. Nature 460: 1127-1131.

De Keyser J, Zeinstra E, Wilczak N (2004). Astrocytic beta2adrenergic receptors and multiple sclerosis. Neurobiol Dis 15: 331-339.
Dennis KE, Levitt P (2005). Regional expression of brain derived neurotrophic factor (BDNF) is correlated with dynamic patterns of promoter methylation in the developing mouse forebrain. Brain Res Mol Brain Res 140: 1-9.

Dutton RC, Maurer AJ, Sonner JM, Fanselow MS, Laster MJ, Eger 2nd EI (2001). The concentration of isoflurane required to suppress learning depends on the type of learning. Anesthesiology 94: 514-519.

Dykman RA, Ackerman PT, Newton JE (1997). Posttraumatic stress disorder: a sensitization reaction. Integr Physiol Behav Sci 32: 9-18.

Fanselow MS, Bolles RC (1979). Naloxone and shock-elicited freezing in the rat. J Comp Physiol Psychol 93: 736-744.

Fanselow MS, Lester LS (1988). A functional behavioristic approach to aversively motivated behavior: predatory imminence as a determinant of the topography of defensive behavior. In: Bolles RC, Beecher MD (eds). Evolution and Learning. Hillsdale: New Jersey. pp 185-211.

Flavell SW, Greenberg ME (2008). Signaling mechanisms linking neuronal activity to gene expression and plasticity of the nervous system. Annu Rev Neurosci 31: 563-590.

Galvez R, Mesches MH, McGaugh JL (1996). Norepinephrine release in the amygdala in response to footshock stimulation. Neurobiol Learn Mem 66: 253-257.

Ge H, Walhout AJ, Vidal M (2003). Integrating 'omic' information: a bridge between genomics and systems biology. Trends Genet 19: $551-560$.

Huang DW, Sherman BT, Tan Q, Collins JR, Alvord WG, Roayaei J et al (2007). DAVID gene functional classification tool: a novel biological module-centric algorithm to functionally analyze large gene list. Genome Biol 8: R183.

Keeley MB, Wood MA, Isiegas C, Stein J, Hellman K, Hannenhalli S et al (2006). Differential transcriptional response to nonassociative and associative components of classical fear conditioning in the amygdala and hippocampus. Learn Mem 13: 135-142.

Lamprecht R, Dracheva S, Assoun S, Ledoux JE (2009). Fear conditioning induces distinct patterns of gene expression in lateral amygdala. Genes Brain Behav 8: 735-743.

Loepke AW, Istaphanous GK, McAuliffe 3rd JJ, Miles L, Hughes EA, McCann JC et al (2009). The effects of neonatal isoflurane exposure in mice on brain cell viability, adult behavior, learning, and memory. Anesth Analg 108: 90-104.

Lubin FD, Roth TL, Sweatt JD (2008). Epigenetic regulation of BDNF gene transcription in the consolidation of fear memory. J Neurosci 28: 10576-10586.

Maier SF, Grahn RE, Kalman BA, Sutton LC, Wiertelak EP, Watkins LR (1993). The role of the amygdala and dorsal raphe nucleus in mediating the behavioral consequences of inescapable shock. Behav Neurosci 107: 377-388.

McCool BA, Farroni JS (2001). Subunit composition of strychninesensitive glycine receptors expressed by adult rat basolateral amygdala neurons. Eur J Neurosci 14: 1082-1090.

Mei B, Li C, Dong S, Jiang CH, Wang H, Hu Y (2005). Distinct gene expression profiles in hippocampus and amygdala after fear conditioning. Brain Res Bull 67: 1-12.

Muotri AR, Chu VT, Marchetto MC, Deng W, Moran JV, Gage FH (2005). Somatic mosaicism in neuronal precursor cells mediated by L1 retrotransposition. Nature 435: 903-910.

Oldham MC, Konopka G, Iwamoto K, Langfelder P, Kato T, Horvath S et al (2008). Functional organization of the transcriptome in human brain. Nat Neurosci 11: 1271-1282.

Ponomarev I, Maiya R, Harnett MT, Schafer GL, Ryabinin AE, Blednov YA et al (2006). Transcriptional signatures of cellular plasticity in mice lacking the alphal subunit of GABAA receptors. J Neurosci 26: 5673-5683.

Rampil IJ, Moller DH, Bell AH (2006). Isoflurane modulates genomic expression in rat amygdala. Anesth Analg 102: 1431-1438.

Ranft A, Kurz J, Deuringer M, Haseneder R, Dodt HU, Zieglgänsberger W et al (2004). Isoflurane modulates glutama- 
tergic and GABAergic neurotransmission in the amygdala. Eur J Neurosci 20: 1276-1280.

Rau V, DeCola JP, Fanselow MS (2005). Stress-induced enhancement of fear learning: an animal model of posttraumatic stress disorder. Neurosci Biobehav Rev 29: 1207-1223.

Rau V, Fanselow MS (2009). Exposure to a stressor produces a long lasting enhancement of fear learning in rats. Stress 12: 125-133.

Rau V, Oh I, Laster M, Eger 2nd EI, Fanselow M (2009). Isoflurane suppresses stress-enhanced fear learning in a rodent model of post-traumatic stress disorder. Anesthesiology 110: 487-495.

Reijmers LG, Perkins BL, Matsuo N, Mayford M (2007). Localization of a stable neural correlate of associative memory. Science 317: 1230-1233.

Roozendaal B, McEwen BS, Chattarji S (2009). Stress, memory and the amygdala. Nat Rev Neurosci 10: 423-433.

Rosen JB, Schulkin J (1998). From normal fear to pathological anxiety. Psychol Rev 105: 325-350.

Roth TL, Sweatt JD (2009). Regulation of chromatin structure in memory formation. Curr Opin Neurobiol 19: 336-342.

Shin LM, Liberzon I (2009). The neurocircuitry of fear, stress, and anxiety disorders. Neuropsychopharmacology 35: 169-191.
Slotkin RK, Martienssen R (2007). Transposable elements and the epigenetic regulation of the genome. Nat Rev Genet 8: 272-285.

Smith MA, Weiss SR, Berry RL, Zhang LX, Clark M, Massenburg G et al (1997). Amygdala-kindled seizures increase the expression of corticotropin-releasing factor (CRF) and CRF-binding protein in GABAergic interneurons of the dentate hilus. Brain Res 745: 248-256.

Sosulina L, Meis S, Seifert G, Steinhäuser C, Pape HC (2006). Classification of projection neurons and interneurons in the rat lateral amygdala based upon cluster analysis. Mol Cell Neurosci 33: $57-67$.

Storey JD (2002). A direct approach to false discovery rates. J Roy Stat Soc B 64: 479-498.

Sugino K, Hempel CM, Miller MN, Hattox AM, Shapiro P, Wu C et al (2006). Molecular taxonomy of major neuronal classes in the adult mouse forebrain. Nat Neurosci 9: 99-107.

Volterra A, Meldolesi J (2005). Astrocytes, from brain glue to communication elements: the revolution continues. Nat Rev Neurosci 6: 626-640.

Waddell J, Bangasser DA, Shors TJ (2008). The basolateral nucleus of the amygdala is necessary to induce the opposing effects of stressful experience on learning in males and females. J Neurosci 28: 5290-5294.

Supplementary Information accompanies the paper on the Neuropsychopharmacology website (http://www.nature.com/npp) 